Original Research Article

\title{
Analysis of medication errors in medicine ward of medical college teaching hospital, Mandya
}

\author{
Supriya K. H. ${ }^{1}$, Shashikumar N. S. ${ }^{1}$, Prakash G. M. ${ }^{2}$, Rajeshwari N. ${ }^{1}$
}

\begin{abstract}
${ }^{1}$ Department of Pharmacology,
${ }^{2}$ Department of General Medicine, Mandya Institute of Medical Sciences, Mandya, Karnataka, India
\end{abstract}

Received: 17 October 2019 Accepted: 18 November 2019

\section{*Correspondence to: \\ Dr. Shashikumar N. S., \\ Email: shashikumar.boss@ gmail.com}

Copyright: (C) the author(s), publisher and licensee Medip Academy. This is an openaccess article distributed under the terms of the Creative Commons Attribution NonCommercial License, which permits unrestricted noncommercial use, distribution, and reproduction in any medium, provided the original work is properly cited.

\begin{abstract}
Background: Medication errors are one of the most common types of medical error that is seen in hospitalised patients. Since medication error is one of the growing concerns of healthcare issue and have implications on patient safety, the purpose of this study was to identify medication error and in turn would help to create awareness among healthcare professionals and provide safety to the patients.
\end{abstract}

Methods: Study was initiated after obtaining approval from the Institutional Ethics Committee. The study was done for a period of 1 year between June 2016 and May 2017. Clinical data and data regarding the medication that was prescribed, transcribed, dispensed, administered was collected and was analysed for various types of medication errors during the different stages of medication use process.

Results: A total of 351 subjects were recruited in the study for assessing medication error. About 2,283 drugs were prescribed among 351 patients. Maximum number of drugs was administered through parenteral route $(50.3 \%)$. Medication error was most common during the prescribing stage $(51.4 \%)$ followed by transcribing stage $(39.1 \%)$, administration stage $(6.9 \%)$, and dispensing stage $(2.6 \%)$. On an average 6 drugs were prescribed per patient. Total of about 5411 errors have occurred out of which $98.8 \%$ of errors were preventable, $1.2 \%$ of errors were non-preventable. Cardiovascular system $(21.9 \%)$ and endocrine and renal system $(21.9 \%)$ was commonly affected due to the medication errors.

Conclusions: Medication errors are one of the commonest problems of the healthcare system should be identified and documented and their causes should be studied in order to develop systems that minimize the recurrence.

Keywords: Prescribing, Transcribing, Dispensing, Administration

\section{INTRODUCTION}

Medication errors (ME) are one of the most common types of medical error that is seen in hospitalised patients. ${ }^{1}$ It is an episode that occurs due to the use of medication, which has to be prevented by using effective control systems. ${ }^{2}$ Number of iatrogenic injuries among patients admitted in the hospital is being increasing leading to economic burden due to the MEs. ${ }^{3}$

ME is defined as "any preventable event that may cause or lead to inappropriate medication use or patient harm, while medication is in the control of healthcare professional, patient or consumer" by the National Coordinating Council for ME Reporting and Prevention. ME is the $3^{\text {rd }}$ most frequent sentinel events $(11.4 \%){ }^{4}$
Different steps involved in ME are prescribing medication, transcribing the order, sending order to pharmacy, dispensing medication, administering medication, documenting the administered medication and assessing its effect on patient. Each one of these steps has potential to cause ME. ${ }^{2}$ ME should be identified and documented and their causes should be studied in order to develop systems that minimize recurrences. ${ }^{4}$

Providing the right medicine to right people at right time is a central priority of health care. The way to ensure this is through the effective implementation of World Health Organization recommendation on rational drug policies. ${ }^{5}$ There are many identifiable factors that can lead to ME such as, insufficient knowledge about drug therapy like, age, impaired renal function and drug allergy, necessity 
for drug dose calculation, knowledge about drug formulation, and medication name for prescribing. ${ }^{2}$

There are various methods used to measure ME like, anonymous self-reports, questionnaires, incident voluntary reporting, critical incident technique, chart review, computer assisted monitoring, disguised direct observation. ${ }^{6} \mathrm{ME}$ are commonly seen among elderly with impaired cognitive function, low literacy or when plenty of high-risk medications are prescribed. ${ }^{7}$ In hospital inpatient ward medication administration error (MAE) is one of the commonest and preventable types of adverse event. ${ }^{8}$ Reporting ME is one of the major issues in healthcare system and prevention of ME is closely related to accurate reporting of ME. ${ }^{9}$

Appropriate medication history is one of the most important steps towards preventing prescription error and is useful in detecting drug related pathology. ${ }^{10}$ Serious medical errors can be prevented by using computerized physician order entry (CPOE) systems which has impact on safety and quality of drug management. ${ }^{11}$ MAE can be prevented by Bar-coded medication administration and ensuring the patient identification, voluntary reporting, direct observation, manual chart review and computerized techniques. ${ }^{8}$ Nursing care is one of the important aspects in prevention of ME. ${ }^{12}$ Pharmacist participation in medical rounds reduces the frequency of $\mathrm{ME}$ as well as their duration once they are identified. ${ }^{13}$

Error is the failure of a planned action to be completed as intended (error of execution) or the use of a wrong plan to achieve an aim (error of planning). It can be an act of commission or an act of omission. Though ME are common during medical practice it is preventable if appropriate measures are taken. ${ }^{14}$ Since ME is one of the growing concerns of healthcare issue and has implications on patient safety, the purpose of this study was to identify and help in preventing errors which has got socioeconomic and health impact. Study on ME would help to create awareness among healthcare professionals and provide safety to the patients. Hence this study is being done to assess ME in medicine ward and their effect on different systems of the body.

\section{METHODS}

Ethical clearance was obtained from the Institutional Ethics Committee before commencing the study. The materials for the present study were collected from inpatient medication chart of all the patients admitted in medicine ward of teaching hospital under Medicine department, Mandya Institute of Medical Sciences, Mandya, Karnataka. Study design was prospective study. Study was conducted for a period of one year from the month of June 2016 to May 2017. 351 patient medication charts of patients admitted in medicine ward were analysed in the study.

\section{Method of collection of data}

Written consent was taken before starting the study. Clinical data regarding age, sex, IP no., date of admission and discharge and diagnosis of the patient was collected from inpatient records. Data regarding the medication that has been prescribed, transcribed, dispensed, administered was collected and was analysed for various types of ME. Patients were assessed for effects of ME on different systems. Patient was followed till discharge from the hospital. All inpatient admitted to medicine ward. All medico-legal cases, cases discharged against medical advice, incomplete records were excluded from the study.

\section{Statistical analysis}

Data was entered into Microsoft Excel and analysis were done using the statistical package for social sciences (SPSS) for Windows software (version 20.0; SPSS). Descriptive statistics was used to analyse the data. Percentage and mean was used to give the results on ME during different stages of medication use. Percentage was also used to represent the effects of ME on different systems.

\section{RESULTS}

The study was conducted for a period of 1 year between June 2016 and May 2017. A total of 351 subjects were recruited in the study for assessing ME. Out of 351 patients, 230 patients were females and 121 were males. About 2,283 drugs were prescribed among 351 patients. Out of 2,283 drugs that were prescribed, 690 drugs were prescribed by generic name and most of them were prescribed by brand name (1593). On an average 6 drugs were prescribed per patient (Table 1).

Table 1: Demographic data.

\begin{tabular}{|ll|}
\hline Variable & N $(\%)$ \\
\hline Gender & \\
\hline Male & $121(34.5)$ \\
\hline Female & $230(65.5)$ \\
\hline Total patients & 351 \\
\hline Total no. of drugs prescribed & 2283 \\
\hline Drugs prescribed by generic name & $690(30.2)$ \\
\hline Drugs prescribed by brand name & $1593(69.8)$ \\
\hline Average drug prescribed per patient & 6 \\
\hline
\end{tabular}

Most of the patients (34.2\%) who were assessed for ME in the present study were between the age group of 40-60 years i.e., the middle age group (Table 2). The commonest cause of admission to the hospital among the study population was infectious disease (29.9\%) i.e., fever due to malaria, typhoid, brucellosis or of viral etiology, followed by respiratory diseases, central nervous system disease, cardiovascular diseases, haematological diseases, endocrine disease, 
gastrointestinal tract (GIT) diseases, hepatobiliary disease and musculoskeletal diseases (Figure 1).

Table 2: Distribution of study subjects according to their age.

\begin{tabular}{|lll|}
\hline Age group (years) & N & $\%$ \\
\hline$<\mathbf{2 0}$ & 19 & 5.4 \\
\hline $\mathbf{2 0 - 4 0}$ & 92 & 26.2 \\
\hline $\mathbf{4 0 - 6 0}$ & 120 & 34.2 \\
\hline $\mathbf{6 0 - 8 0}$ & 112 & 31.9 \\
\hline$>\mathbf{8 0}$ & 8 & 2.3 \\
\hline Total & 351 & 100 \\
\hline
\end{tabular}

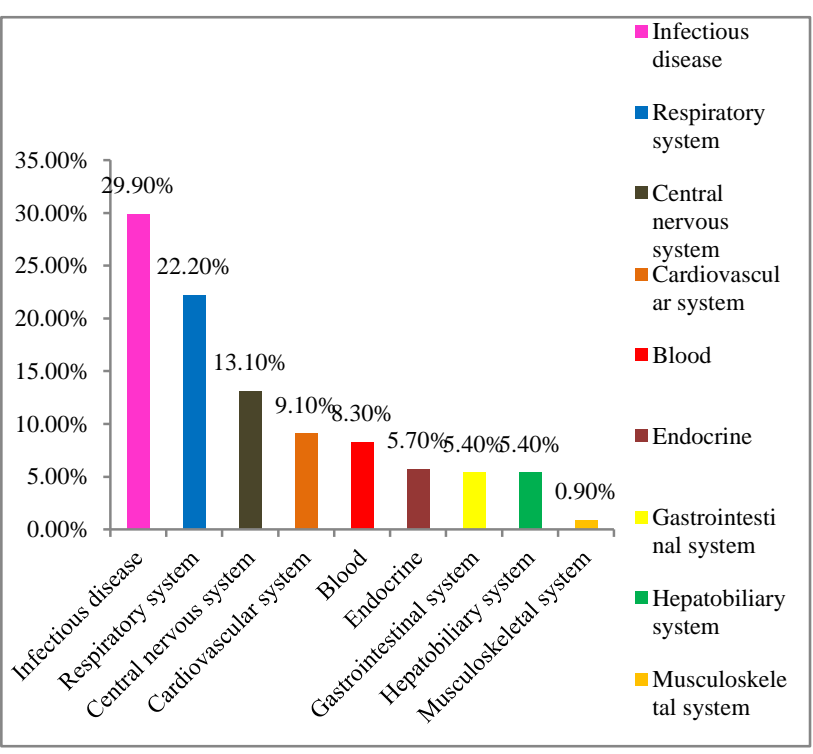

Figure 1: Percentage of patients presented with different diseases affecting different systems.

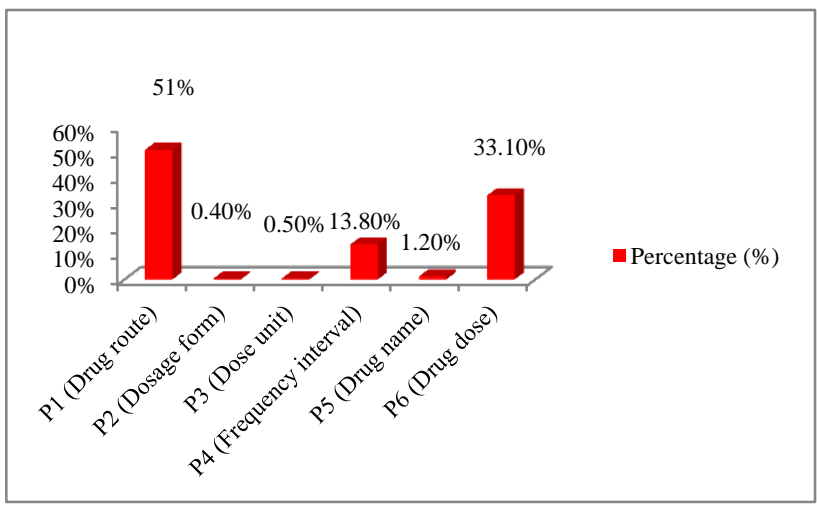

Figure 2: Percentage of medication error during drug prescribing stage.

ME were seen in almost all the prescriptions that were prescribed among 351 patients. About 2745 drugs that were prescribed had prescribing errors, most of the prescribing error was $\mathrm{P} 1$ type $(51 \%)$ i.e., the drug route was either not mentioned or wrongly written followed by P6 type (33.1\%) i.e., drug dose was not mentioned or was wrongly written, $\mathrm{P} 4$ (frequency interval) type (13.8\%), P5 (drug name) type (1.2\%), P2 (dosage form) type (0.4\%), P3 (dose unit) type $(0.5 \%)$ (Figure 2$)$.

Transcribing error is the one that occurs while writing down the orders that is being given by the prescribing physician. In this study transcribing error was more common in the transcribing stage 1 (92.9\%). Total of about 2091 transcribing error was seen in both the stages of transcribing drugs. In stage 1, transcribing error was commonly seen with frequency interval (37.6\%) and route $(37.4 \%)$ of drug administration. In stage 2, transcribing error was commonly seen due to fewer requests of drugs $(55.7 \%)$ (Figure 3 ).

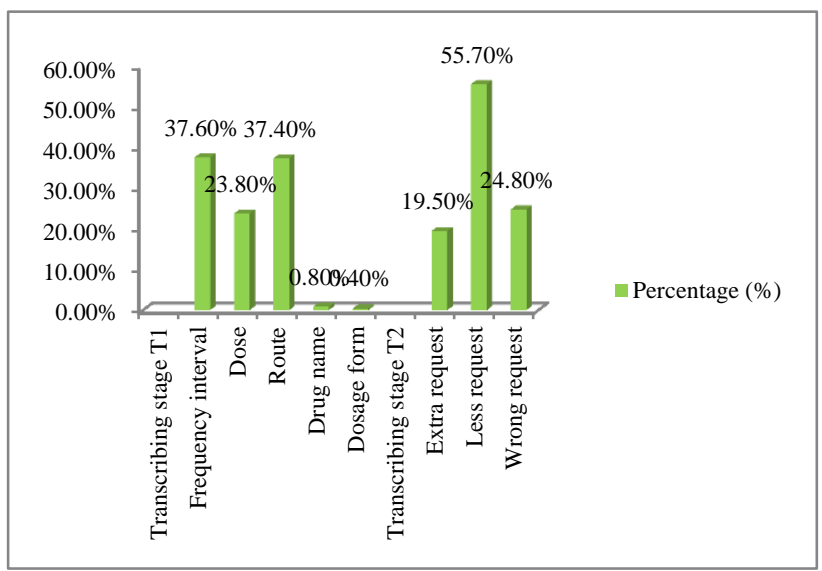

Figure 3: Percentage of medication error during drug transcribing stage.

Drug administration error commonly occurs due to nursing staff, who are involved in drug administration to the patients. Total of about 365 errors were seen during the stage of administering drugs to the patient. Administration error was common due to omission of drug i.e., medication not given $(47.1 \%)$ to the patient (Figure 4).

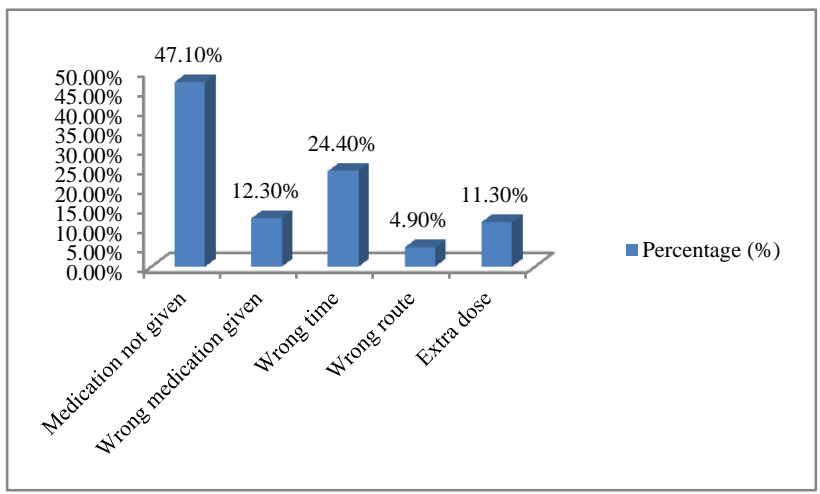

Figure 4: Percentage of medication error during drug administering stage.

About 138 errors have occurred while dispensing the drugs during the study period. Most of the time dispensing error was commonly due to failure to send medicine to ward at appropriate time (50\%) (Figure 5). 


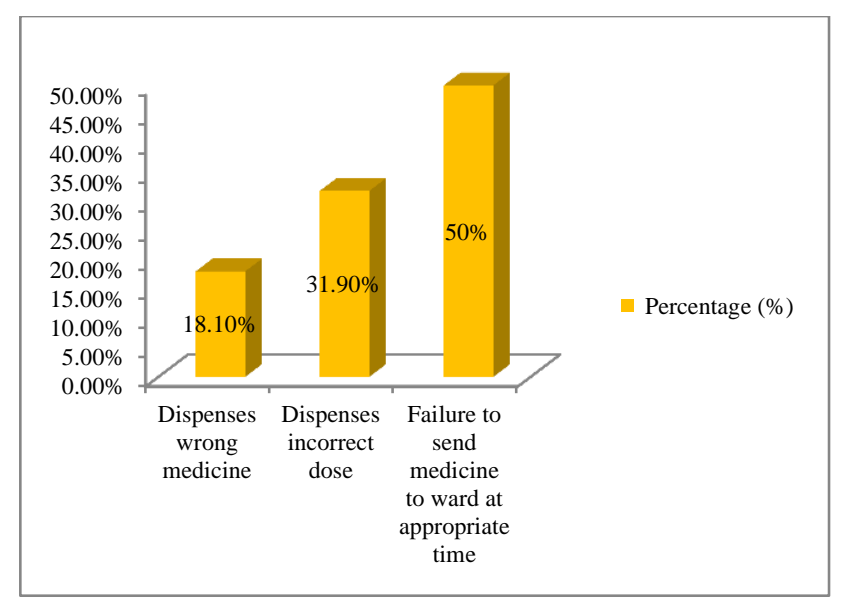

Figure 5: Percentage of medication error during drug dispensing stage.

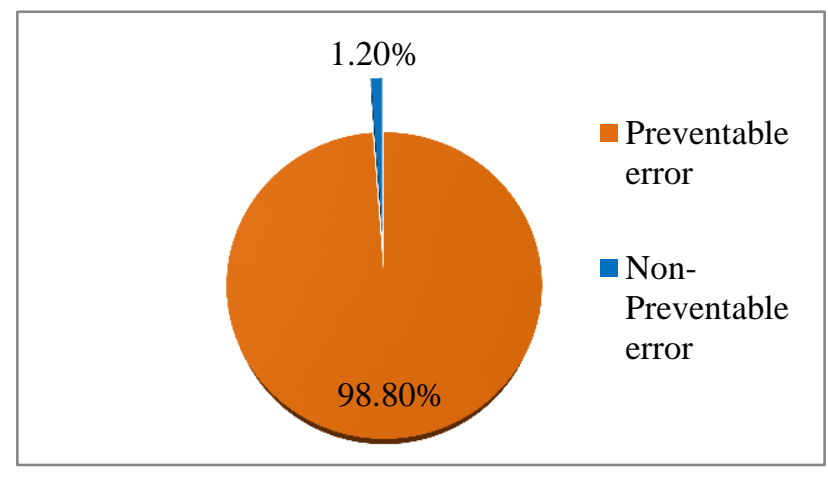

Figure 6: Percentage of different types of medication errors.

Table 3: Different types of medication errors during different stages of medication use process.

\begin{tabular}{|lll|}
\hline Stages of medication use & No. of errors & $\%$ \\
\hline Drug prescribing stage & 2745 & 51.4 \\
\hline Drug transcribing stage & 2091 & 39.1 \\
\hline Drug administration stage & 365 & 6.9 \\
\hline Drug dispensing stage & 138 & 2.6 \\
\hline Total & 5339 & 100 \\
\hline
\end{tabular}

Total of about 5411 errors [Figure 6] have occurred during our study period out of which $98.8 \%$ of errors were preventable type, which was commonly seen due to error that occurred during prescribing, administering, transcribing or dispensing of the drugs. In this study it was found that $\mathrm{ME}$ was most common during the prescribing of medication $(51.4 \%)$ and least common during dispensing medication (2.6\%) [Table 3]. About $1.2 \%$ of errors were non-preventable which is mainly due to side effects of the drugs that are used in treatment of the patient, which cannot be prevented.

ME can lead to adverse effect on different systems of patients, in this study cardiovascular system $(21.9 \%)$ and endocrine and renal system (21.9\%) was commonly affected due to the ME, followed by respiratory system (16.1\%), GIT (13.9\%), haematological system (11.7\%), etc. (Figure 7).

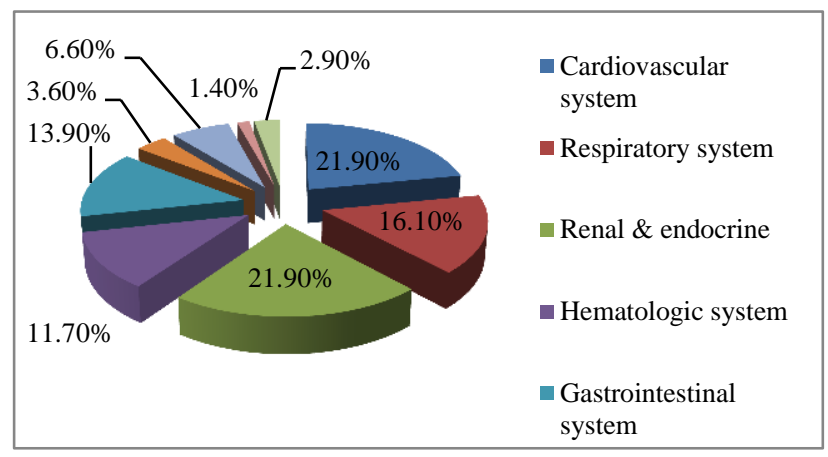

Figure 7: Percentage of different systems affected due to medication errors.

\section{DISCUSSION}

Iatrogenic harm is one of the important public health problems which can lead to loss of economy and $\mathrm{ME}$ is one of the iatrogenic harm caused by the health care professionals. ${ }^{15}$ MEs are one of the serious problems in the health care system which can lead to morbidity and mortality. ${ }^{3} \mathrm{ME}$ is an episode that occurs due to the use of medication that should be prevented through effective control systems. ${ }^{2}$

ME can occur at different phases of the medication use process from prescription by the physician, to dispensing by the pharmacist, or administration by the nurse. ME may be due to poor medication labelling, miscommunication among clinicians, lack of verification, an incomplete medication prescription or inadequate staffing. ${ }^{12}$ Medication safety is a part of quality of care and patient safety. ${ }^{16}$

In this study about 2,283 drugs were prescribed among 351 prescriptions. Out of 2283 drugs, 690 drugs were prescribed by generic name and 1593 drugs were prescribed by brand name [Table1]. Though the drugs have to be prescribed by generic name most of the drugs in this study were prescribed by brand name which may be because it is easier to remember and most of the combination drugs are available with brand name.

In this study about $65.5 \%$ of patients were females and $34.5 \%$ of them were males [Table1], $34.2 \%$ of patients belonged to $40-60$ years and $31.9 \%$ of patients belonged to 60-80 years age group [Table 2]. In a study done by Khavane et al, $77.4 \%$ patients were male and $22.6 \%$ were females and about $38.5 \%$ of them were in the age group of 40 to 60 years, which when compared to our study had more number of male patients but the age group was similar to the present study. ${ }^{2}$

About 2745 prescribing errors were seen among 351 prescriptions. Commonest type of prescribing error was 
P1 type (51\%) i.e., drug route was either not mentioned or was wrongly written and P6 type $(33.1 \%)$ i.e., drug dose was not written or was mentioned wrongly, P2 type $(0.4 \%)$ i.e., dosage form was least common [Figure 2]. In a study conducted by Das et al about 1632 prescription errors were present, prescription errors were most commonly due to dosage form $(37.9 \%)$ and least common due to route of drug administration ( $0.4 \%)$, which is opposite to this study where drug route was the common type of prescribing error and dosage form was the least common type of prescribing error. ${ }^{17}$ Prescribing faults and prescription errors are major problems among ME. Although they are rarely fatal they can affect patients' safety and quality of healthcare. Prescription errors are typically events that derive from slips, lapses, or mistakes. ${ }^{18}$

Total of about 2091 types of transcribing errors were seen in both the stages of transcribing (stage 1 and stage 2) in the present study. About $92.9 \%$ of errors were seen in transcribing stage 1 i.e., fault in frequency interval $(37.6 \%)$, route $(37.4 \%)$, dose $(23.8 \%)$, drug name $(0.8 \%)$ and dosage form $(0.4 \%)$. About $7.1 \%$ of errors were seen during transcribing stage 2 i.e., less request $(55.7 \%)$, wrong request $(24.8 \%)$ and extra request $(19.5 \%)$ [Figure 3]. Many ME occur during transcription process, mainly due to the similar drug name or sound alike drugs. Transcription is the term used to describe the complex set of tasks involved in interpreting and processing orders. Transcription error can be reduced by using computerized provider order entry systems which eliminates the handwritten prescriptions. ${ }^{19}$

Medication administration is one of the commonest risk areas and in order to prevent harm and safeguard the patients safety, drug has to be administered properly. Some of the most common factors that lead to MAE are failure to check the patient's identification prior to administration, the storage of similar preparations in similar areas, and environmental factors like nurse interruptions while undertaking a drug round. Also inappropriate documentation and poor communication during change of shifts in the hospitals can contribute to MAE. ${ }^{8}$

About 365 (6.9\%) drug administration error was seen in the present study, administration error was common due to not giving the medication $(47.1 \%)$ and least common was wrong route $(4.9 \%)$ (Figure 4$)$. In a study conducted by Sarah et al $27.6 \%$ of administration errors were seen, $72.6 \%$ of MAE was due to administering drug at wrong time, $14 \%$ were due to not giving the medication, which shows that administration error was more common than our study. ${ }^{20}$ Most common cause of MAE in our study was due to not administering the medication whereas in a study conducted by Sarah et al commonest cause was due to administration of drug at wrong time.

Since large number of medicines will be dispensed per day in hospital pharmacy there is a tendency for dispensing error to occur. Most of the time drug dispensed by pharmacy is checked by the nursing staff in the ward thereby only few of the errors reach the patient. Bar code technology can prevent dispensing errors to certain extent. ${ }^{21}$ About 138 errors were seen during the dispensing stage of ME in the present study, commonest type of dispensing error was due to dispensing drug to the ward at inappropriate time $(50 \%)$ followed by dispensing incorrect dose $(31.9 \%)$ and dispensing wrong medicine (18.1\%) (Figure 5). Dispensing error can be prevented if the quality of pharmacy care is assured by the pharmacist. $^{22}$

Total of about $5406 \mathrm{ME}$ were seen out of that $98.8 \%$ were preventable type of error and $1.2 \%$ were nonpreventable error (Figure 6). In a study conducted by Majd et al $76 \%$ of errors were classified as preventable error which is lesser than this study. ${ }^{9}$ This shows that most of the errors in the present study are due to human faults rather than the adverse effect caused by the drug and is preventable if appropriate measures are taken.

In the present study maximum number of errors was seen during the stage of prescribing $(51.4 \%)$, followed by transcribing stage $(39.1 \%)$, drug administration stage (6.9\%) and dispensing stage (2.6\%) (Table 3). According to the study done by Asad et al prescribing error was seen in $53.9 \%$ which is almost similar to the present study, administering error was seen in $34.8 \%$, which is much more than what is seen in the present study, dispensing error $(0.6 \%)$ was the least common similar to our study and transcribing error was seen in $10.7 \%$ of cases. ${ }^{23}$ Most of the errors occurred during prescribing stage, since drug prescriptions are mostly written by the junior doctors who doesn't have much knowledge about drug dose, route of administration and frequency interval. Hence it is necessary to train the junior doctors about writing prescription to the patient before they start with their internship. Cardiovascular $(21.9 \%)$ and renal and endocrine $(21.9 \%$ ) (Figure 7) were the commonest systems that were affected due to the ME that occurred during the present study. ME have led to hypotension, tachycardia, bradycardia, electrocardiography variations, hypoglycaemia, electrolyte imbalance, drug induced gastritis in the present study.

Patient related factors are also important in clinical conditions like reduced renal and hepatic function or a history of allergy, the dose and frequency of the drug has to be altered in these conditions. ${ }^{24}$ Hence prescribing without considering the patient's clinical status, failure to communicate essential information and transcription errors will lead to prescribing errors. ${ }^{1}$

Errors may be more frequently made by junior members of staff who work under stressful condition and also inadequate knowledge or training about use of medication may be the reason for prescribing faults. ${ }^{24}$ Inadequate feedback control or lack of cooperation between doctor and nurses can cause error leading to 
adverse effect. When errors are minimized and when the prescriber actively endeavours to achieve better prescribing, appropriate prescribing can occur. ${ }^{24}$

Reporting of error should be made as easy as possible (forms should be accessible and straightforward), and also feedback should be given regarding the report that is being submitted, and educate about all aspects of the ME reporting process at their hospitals. ${ }^{25}$ Though the ME have been detected during different stages of medication use and its effect on different systems is being analysed, there are certain limitations in this study since study was done only in the Medicine ward, the errors that are detected cannot be generalised to the whole hospital, the results are confined only to the Medicine ward.

Since maximum number of errors were seen during prescribing and transcribing stage, hence good training of the healthcare professionals is required and proper guidelines has to be followed in order to prevent the occurrence of error and provide safety to the patients.

\section{CONCLUSION}

ME are one of the commonest problems faced by the healthcare system which can significantly harm the patient. It is said that errors can occur in any of the systems but it is important to find the cause and attempt to prevent the occurrence of errors. As the access to the medical care is being increasing in recent years, the susceptibility for the occurrence of ME is also becoming common. The goal of any drug therapy is to achieve defined therapeutic outcome that minimizes the risk and improves the quality of life of patient. The error can occur due to any of the healthcare professional at any time of the health care process. In order to reduce the occurrence of errors, it is necessary for health institutions to maintain a continuous careful analysis of the errors that occurs effectively.

MEs should be monitored, detected early and should be prevented as much as possible to safeguard the health of the patient. There is also need to improve the quality of programs for monitoring ME. Patient education and participation in their own healthcare decisions should be encouraged. All those who are involved in healthcare should also join hands to solve this gigantic problem of ME.

\section{ACKNOWLEDGEMENTS}

We would like to acknowledge the staff members of Department of Medicine, Mandya Institute of Medical Sciences, Mandya, Karnataka.

Funding: No funding sources Conflict of interest: None declared

Ethical approval: The study was approved by the Institutional Ethics Committee

\section{REFERENCES}

1. Dean B, Schachter M, Vincent C, Barber N. Prescribing errors in hospital inpatients: their incidence and clinical significance. Qual Saf Health Care. 2002;11:340-4.

2. Khavane K, Sanjay S, Shivkumar I, Anil B. Study and evaluation of medication error in a tertiary care teaching hospital-A baseline study. Int J Pharm Pharm Sci. 2012;4(5):587-93.

3. Purushothama RK, Mallesh M. Medication errors: identification, prevention and implementation of safe medication practice in a tertiary care teaching hospital. World J Pharm Pharmaceutical Sci. 2015;4(3):1249-56.

4. Kriti M, Manoj G, Rani W, Shafiqua A. Medication errors: A preventable problem. Indian JClin Practice. 2012;23:17-21.

5. Afroz A, Surabhi G, Saurabh K, Ramgopal. Prescription auditing and drug utilization pattern in a tertiary care teaching hospital of Western UP. Int J Basic Clin Pharmacol. 2012;1(3):184-90.

6. Fatmeh S, Amir ZH. Medication error detection in two major teaching hospitals: What are the types of errors?. J Res Med Sci. 2014;19(7):617-23.

7. Sunil K, Christianne LR, Anuj KD, Courtney C, Alexandra B, Svetlana KE, et al. Effect of a pharmacist intervention on clinically important medication errors after hospital discharge: a randomized controlled trial. J Am Med Dir Assoc. 2010;11:275-83.

8. Senafikish AF, Muluadam AM, Yeshaneh SY. Medication administration error: Magnitude and associated factors among nurses in Ethiopia. BMC Nurs. 2015;14(53):1-8.

9. Majd TM, Kawkab S, Ibrahim ALF. Rate, causes and reporting of medication errors in Jordan: nurses' perspectives. Assistant J Nurs Manag. 2007;15:65970.

10. Richard FJ. Medication errors: the importance of an accurate drug history. $\mathrm{Br} \mathrm{J}$ Clin Pharmacol. 2009;67(6):671-5.

11. Khajouei R, Jaspers MWM. The impact of CPOE Medication systems' design aspects on usability, workflow and medication orders: a systematic review. Iran Methods Inf Med. 2010;49:3-19.

12. Malcolm E, Yisi L. The nine rights of medication administration: an overview. Br J Nurs. 2010;19:3005.

13. Kimberly KS, Michael AF, Gary AN. Pharmacist participation in medical rounds reduces medication errors. Am J Health-Syst Pharm. 2002;59:2089-92.

14. Vesile U, Sevinc T, Nalan A. Medication errors: Perspectives of newly graduated and experienced nurses. Int J Nurs Practice. 2012;18:317-24.

15. Alan FM, Craig SW, Jacqueline HS, Mitchell J, Robert H, Papaarangi R. Multimodal system designed to reduce errors in recording and administration of drugs in anaesthesia: prospective randomised clinical evaluation. BMJ. 2011;1-14. 
16. Eija MRT, Ulla VRN. Medication errors in elderly acute care - a systematic review. Scand J Caring Sci. 2014;28:12-28.

17. Das DGS, Mario BR, Bryony DF, Adriano MMR, Leni MA, Joaquim ACM. Concomitant prescribing and dispensing errors at a Brazilian hospital: a descriptive study. CLINICS. 2011;66(10):1691-7.

18. Sara G, Matthew R, Liesbeth D, Bryony D F. Measuring the severity of prescribing errors: a systematic review. Drug Saf. 2013;36:1151-7.

19. Philip A, Julie AW, Lyle JB, Linda RC. Preventing medication errors. Committee on Identifying and Preventing Medication Errors. Quality Chasm Series. 2007; 75-6.

20. Sarah B, Brigitte S, Florence G, Thibaut C, Patrice P, Pierre D. Evaluation of drug administration errors in a teaching hospital. BMC Health Services Res. 2012;12(60):1-8.

21. Eric GP, Jennifer LC, William C, Nirali P, Erica F, Jeffrey MR, et al. Medication dispensing errors and potential adverse drug events before and after implementing bar code technology in the pharmacy. Ann Intern Med. 2006;145:426-34.
22. Ka-Chun C, Marcel LB, Peter AGM. Medication errors the importance of safe dispensing. $\mathrm{Br} \mathrm{J}$ Clin Pharmacol. 2009;67(6):676-80.

23. Asad EP, Terri LW, Arthur BS, Brian LE. A Prospective observational study of medication errors in a tertiary care emergency department. Ann Emerg Med. 2009;20:1-5.

24. Giampaolo PV, Pietro M. Medication errors: prescribing faults and prescribing errors. Br J Clin Pharmacol. 2009;67(6):624-8.

25. Nicole H, Neil MK, Ingrid S, Mark F. Identifying, understanding and overcoming barriers to medication error reporting in hospitals: a focus group study. BMJ Qual Saf. 2012;21:361-8.

Cite this article as: Supriya KH, Shashikumar NS, Prakash GM, Rajeshwari N. Analysis of medication errors in medicine ward of medical college teaching hospital, Mandya. Int J Basic Clin Pharmacol 2019;8:2741-7. 